\title{
Metodología para la Evaluación de las Tecnologías de Información en la Docencia de Proyectos de Ingeniería
}

\author{
Manuel F. Marey, Carlos Amiama y Carlos J. Álvarez \\ Universidad de Santiago de Compostela, Departamento de Ingeniería Agroforestal, \\ Escuela Politécnica Superior, Campus Universitario, 27002 Lugo-España \\ (e-mail: carlosjose.alvarez@usc.es)
}

\section{Resumen}

En este trabajo se presentan las características principales del proyecto de e-aula desarrollado en la Universidad de Santiago de Compostela-España y se valida una metodología para la evaluación de este tipo de tecnologías de apoyo a la docencia. Se analizan estadísticamente los resultados obtenidos en la valoración realizada por los alumnos de una aplicación desarrollada para docencia virtual. Se relacionan los resultados con las calificaciones obtenidas por los alumnos en la asignatura, mediante el empleo de técnicas de análisis de correlación multivariante y análisis factorial discriminante. Se concluye acerca de la utilidad de estas técnicas para realizar inferencia de resultados futuros, a partir de valoraciones previas realizadas por los alumnos.

Palabras clave: e-aula, análisis factorial discriminante, TIC, estrategias de enseñanza

\section{Methodology for the Evaluation of Information Technology in the Teaching of Engineering Projects}

\begin{abstract}
In this work the basic characteristics of an e-learning project done at the University of Santiago de Compostela-Spain are presented, and a methodology for the evaluation of this type of teaching support technology is validated. The results obtained in an evaluation performed by the students on an application developed for virtual teaching, are statistically analyzed. The results are related to the qualifications obtained by the students, using multivariate and correlation analysis techniques, and discriminate factorial analysis. Based on the results, conclusions are drawn about the usefulness of these techniques to make inference of future results, based on previous assessments made by students.
\end{abstract}

Keywords: e-learning, ex-post evaluation, factorial discriminator analysis, TIC, teaching strategies 


\section{INTRODUCCIÓN}

La utilización de las Tecnologías de la Información y Comunicaciones (TIC's) como ayuda en la docencia ha venido desarrollándose y perfeccionándose durante las últimas décadas, aunque su aplicación a la docencia universitarias es mas limitada (Nieto y Rodriguez, 2007). La actitud del profesorado hacia la utilización de estas tecnologías también evoluciona positivamente en los últimos años (Manassero et al., 2004), siendo cada vez mayor el número de profesores universitarios que las incluyen en su docencia, con mayor o menor grado de complementariedad con la docencia tradicional. De cualquier modo es indiscutible que la adopción de estas tecnologías exige formación en la utilización de las mismas (Marín, 2007), esfuerzo que es dificultoso y plantea reacciones de rechazo (Hernández, 2006), dado que muchos docentes no ven claros sus beneficios (García y Pinto, 2005).

Actualmente, existe una significativa tendencia en las universidades europeas para dotarse de sistemas informáticos que faciliten o complementen la enseñanza tradicional (Bautista et al., 2006), en un intento dentro de la convergencia educativa europea de acceder a un mayor número de alumnos y de dotarles de mejor formación en un contexto de competencia para la captación de alumnos (Bosco, 2005). Muchos autores consideran fundamental la utilización de las TIC's en este desafío (Cebrián, 2004). En las revistas especializados se pueden obtener múltiples referencias de estudios y aplicaciones de estas tecnologías y de sus efectos en diferentes países, por ejemplo Brasil (Maltempi y Rosa, 2006), Colombia (Henao, 2006), Chile (Arancibia, 2006; Salcedo et al., 2006), España (Tejedor y García-Valcárcel, 2006), Estados Unidos (Salas, 2005), México (Cervantes y Barrios, 2005), Perú (Valverde y Rivas, 2005) o Venezuela (Lavilla et al., 2006).

En el presente trabajo se realiza un estudio de caso en España, concretamente en la Escuela Politécnica superior de la Universidad de Santiago de Compostela, en la titulación de Ingenieros Agrónomos, que se imparte desde el 1988, y en la de Ingenieros de Montes, que se imparte desde 1994. Ambas titulaciones se encuentran en un debate intenso en la actualidad frente a su estructuración de acuerdo con el Espacio Europeo de Educación Superior, de acuerdo con la declaración de Bolonia de 1999 (González y Wagenaar, 2003). Concretamente, el presente estudio, se realiza con alumnos que acaban de cursar la materia de proyectos, la cual pretende que los futuros ingenieros desarrollen a partir de los conocimientos que adquieren capacidades para el uso consciente y adecuado de diferentes formas de pensar y tomar decisiones, para lo que deberán de aplicar las características analíticas y sintéticas de la forma más conveniente en cada momento. Por ello los profesores de proyectos definen sus objetivos y pretenden articular la aplicación deliberada de conocimientos adquiridos por los alumnos, desarrollando la conciencia de su facultad creativa (el diseño), del planteamiento sistemático de los problemas (la metodología) y de la toma de decisiones (la gestión).

\section{La percepción de los alumnos/ingenieros}

Desde el curso académicos 2001/02, hasta la actualidad, los profesores de la asignatura de proyectos realizan encuestas a los alumnos, en el momento de comenzar el curso. Estas encuestas presentan dos objetivos, el primero es conocer sus expectativas profesionales como futuros ingenieros y el segundo, conocer lo que demandan de la materia de proyectos, para dar respuesta a esas necesidades. Los resultados ponen de manifiesto, en primer lugar, el temor a la salida al mercado laboral y la consideración de que sus conocimientos no son los suficientes ni los adecuados para alcanzar un puesto de trabajo. En segundo lugar, el conflicto entre alcanzar los conocimientos que se consideran necesarios en su formación y el esfuerzo que están dispuestos a realizar para la superación de la asignatura.

A lo largo de estos años se ha contrastado que el esfuerzo hecho en transmitir esta idea de la importancia de "como hacer las cosas" no ha sido baldía. El esfuerzo por conocer lo que opinaban los alumnos se trasladó también a observar cual es la importancia real del trabajo docente una vez que estos salen al mercado laboral. De este modo, en el año 2003, dos de los profesores de la asignatura de proyectos realizaron un estudio sobre la situación de los ingenieros agrónomos que 
estaban ya ejerciendo (Álvarez et al., 2003). A la pregunta “¿cuales considera las temáticas más importantes en la formación del ingeniero agrónomo?" el resultado fue que son aquellas relacionadas con los proyectos de ingeniería las que alcanzaron las mayores puntuaciones. Estos resultados se vieron confirmados por la encuesta realizada por la dirección de la Escuela Politécnica Superior, en el año 2006, a los titulados de los últimos 5 años. Los resultados de dicho trabajo destacan que la asignatura de proyectos es la más valorada para su formación por parte de los recién titulados.

\section{Situación actual de la docencia y continuidad para el futuro}

Es necesario destacar que desde el curso académico 2002/2003 los profesores que imparten esta materia han realizado un esfuerzo de modificación de las mismas, pasando de un planteamiento tradicional teórico a lo que puede ser un futuro dentro del Espacio Europeo de Educación Superior. Este aspecto totalmente novedoso a nivel nacional, entre todas las Escuelas de Agrónomos y Montes, se añade la potencialidad de las nuevas TIC's, que permiten alcanzar mejores resultados en calidad de la docencia y en la formación del ingeniero.

El esfuerzo de los autores de este trabajo sigue estando en conocer cual debe ser el método de docencia que permita: 1) A los docentes determinar qué es necesario enseñar a los futuros titulados, teniendo en cuenta las restricciones de medios y tiempo de que disponemos. 2) Al alumno ser consciente de la necesidad de aprender y manejar lo que se le está mostrando en clase, y a su vez que observe su posición relativa respecto a los conocimientos necesarios, tanto para superar la asignatura como para incorporarse al mercado laboral. Para ello se han utilizado los recursos TIC.s de la Universidad de Santiago de Compostela.

En los últimos años la USC viene realizando un gran esfuerzo para dar respuesta a la creciente demanda de integración de las Tecnologías de la Información y las Comunicaciones en el proceso de enseñanza-aprendizaje, tanto como elemento complementario a la docencia presencial tradicional, como en la generación de nuevas modalidades de formación síncronas y asíncronas basadas en el uso intensivo de factores y útiles tecnológicos. Así, el incremento en la demanda por parte del personal docente de espacios virtuales para complementar su docencia hace necesario que sus esfuerzos sean reconocidos adecuadamente, lo cual es difícil de llevar a cabo cuándo los recursos generados se encuentran dispersos por toda la red. Como una de las respuestas la estas demandas se creó en diciembre de 2003 el Centro de Tecnologías para el Aprendizaje (CeTA) como un servicio universitario que tiene como finalidad coordinar y apoyar a la integración racional de las tecnologías de la información y las comunicaciones en el proceso de enseñanza y de aprendizaje.

Con el establecimiento del Campus Virtual, la USC ha buscado propiciar actividades formativas que se desarrollen a través de la red, al tiempo que se incorpora a la formación de los estudiantes a generación de habilidades y actitudes relacionadas con el empleo de las TIC, tan importantes en su futura actividad social y profesional. Además, el Campus Virtual pretende facilitar la aplicación de metodologías asociadas a la implantación del Crédito Europeo (ECTS), así como el acceso a los programas de Postgrado, Tercer Ciclo y Formación Continua de la USC a todas aquellas personas que deseen cursarlos.

En el marco de la $4^{\circ}$ convocatoria de proyectos de innovación educativa de la USC, un grupo de profesores de proyectos, coordinados por M.F. Marey, presentó un proyecto de e-aula, denominado "Tecnologías web para la docencia de la materia de proyectos de ingeniería", que fue seleccionado como mejor proyecto del área tecnológica de la Universidad de Santiago de Compostela. Dentro del objetivo global de desarrollar instrumentos que permitan mejorar la calidad de la docencia, calibrándola con un mismo instrumento y sistema de medición por parte del profesorado y el alumnado, se pretende conocer el impacto que ha tenido la utilización de un aula virtual como herramienta de apoyo a la docencia presencial. Se desea evaluar, desde un punto de vista general, la aprobación por parte del alumnado y diferenciar, de acuerdo al resultado obtenido por cada alumno en las pruebas de la asignatura, si se puede elaborar un modelo que permita conocer el resultado académico posterior a través del trabajo con la aplicación virtual. 
Todo ello dentro del concepto innovador del Espacio Europeo de Ecuación Superior el cual se establecerá en España en el curso 2009/2010, donde el planteamiento de enseñanza y formación personal y participativa de los alumnos, puede ser ayudada eficazmente con la utilización de plataformas virtuales que potencien el trabajo de los alumnos (ERT, 1995).

\section{MATERIAL Y MÉTODOS}

\section{La herramienta: la e-aula}

La USC cuenta con una plataforma virtual, denominada Web-CT, a disposición de los docentes que quieran utilizarla para la docencia virtual. Esta herramienta permite configurar los contenidos acorde a las necesidades del docente. Con la experiencia de lo avanzado hasta el momento se considera que la docencia a través de Web-CT contribuye a:

- Coordinar los contenidos de las materias facilitados a los alumnos en las diferentes asignaturas. - Apoyar mutuamente a los docentes entre si en aspectos ligados a la teoría y la práctica de lo que conlleva el ser profesor de ingenieros proyectistas en la actualidad.

- Intercambiar la información da que se dispone en un mismo formato de trabajo y de forma sistematizada.

- Analizar la respuesta obtenida por parte del alumnado, permitiendo conocer cuales son los aspectos de mayor complejidad de compresión, conocer su importancia para la formación y las causas de la dificultad de aprendizaje.

- Seguir de forma individualizada el avance obtenido por cada alumno, a la vez que se conoce el comportamiento conjunto a través de indicadores estadísticos que permiten variar el ritmo del curso, enfatizando en aquellos aspectos que se observen carencias en la formación, y que inciden en el antes mencionado sistema proyecto.

Específicamente, esta herramienta permitirá además:

- Participar de forma individual o como grupo en el desarrollo de la asignatura y de su formación, a través de los diferentes elementos de que dispone el sistema (foro, chat...). Este aspecto es especialmente importante para la asignatura de proyectos en la cual se estableció anteriormente como esencial el trabajo en grupo.

- Disponer de forma centralizada y accesible en tiempo de toda la información teórica de la asignatura, lo que permitirá facilitar y completar la formación aportada en las aulas, al igual que los contenidos prácticos de la misma, que podrán ser llevadas a cabo con mayor dinamismo.

- Formar en el manejo de tecnologías que, sin duda, tendrán gran importancia en su futuro como alumnos y especialmente como profesionales.

- Manejar herramientas informáticas disponibles en la subárea (software específico para la realización de apartados del sistema proyecto), que pueden ser accesibles a través de internet el tiempo que precise para familiarizarse con su manejo.

Además permitirá una mayor interacción entre el profesorado y el alumnado fundamentado en los siguientes aspectos:

Aumentar la motivación del alumnado para afrontar la asignatura y el conjunto de la titulación.

- Mejorar la formación del alumno, mediante el acceso rápido y económico a toda la información necesaria para su formación, al igual que otros contenidos que le exige el mercado.

- Facilitar la enseñanza personalizada y favorecer el feedback profesor y alumno, potenciando la labor tutorial, a través de la red, y el papel del profesor como orientador del aprendizaje.

- Favorecer el autoaprendizaje y la reflexión, competencias de las que debe de disponer un ingeniero.

- Conseguir la consideración del trabajo en equipo desarrollada de manera presencial o virtual a través de la red.

- Gestionar las limitaciones de tiempo y adaptar el trabajo a las condiciones individuales o del grupo. 
Con todos los objetivos anteriores se elaboró una aplicación informática, sobre la plataforma WebCT, denominada "Tecnologías web para la docencia de la materia de proyectos de ingeniería", que está organizada en las siguientes áreas de contenidos:

- Núcleos temáticos: un espacio para cada tema del programa del curso. Incluye textos y lecturas complementarias, guías didácticas, esquemas y resúmenes de los temas, fuentes estadísticas, enlaces a páginas web de interés, ejercicios de autoevaluación, desarrollo de lecciones temáticas "virtuales", realización de prácticas en red, etc.

- Información administrativa: Fundamentalmente fechas de exámenes, revisiones, prácticas... Incluye además información relacionada con la actividad académica (conferencias, charlas, etc.) y con la actividad profesional relacionada con el mundo de los proyectos y la planificación (legislación, convocatorias de ayudas, etc.)

- Recursos comunes compartidos: bases de datos, materiales multimedia, cartografía (temática o general) para la realización de prácticas, al igual que otros recursos que sean de especial interés para el trabajo individual o de grupo. Específicamente en este apartado se encuentran las comunicaciones de los 5 últimos congresos de AEIPRO, clasificadas por áreas temáticas.

- Área de intercambio: en el cual están los foros de debate, las noticias y los eventos de interés, intercambios entre alumnos, intercambios entre profesores, etc.

- Sistemas de tutorías: e-mail, chat, pizarra electrónica, etc. Con la utilidad de poder seguir y coordinar trabajos de curso individuales o colectivos (anteproyectos), y los proyectos fin de carrera.

- Carpeta personal del alumno o del grupo de trabajo.

La pantalla de acceso principal a la aplicación para la titulación de ingenieros agrónomos se muestra en la figura 1

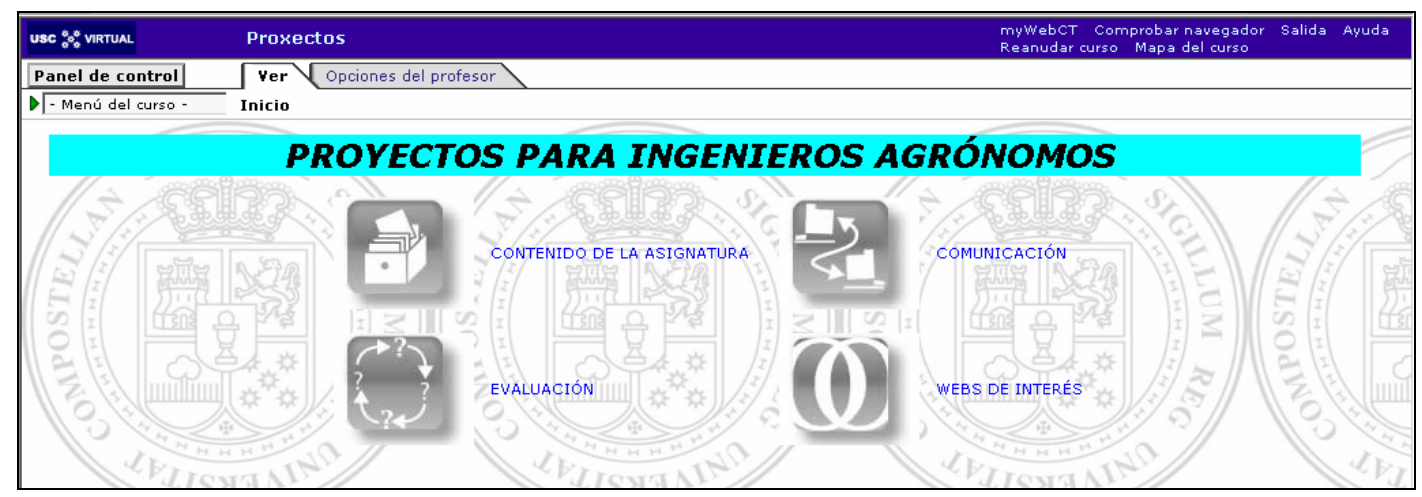

Fig. 1: Pantalla de acceso para los alumnos de ingeniería agronómica

\section{Evaluación de la aplicación}

Tras el esfuerzo realizado y como viene siendo necesario en todos los proyectos que se ponen en marcha, es necesario establecer una metodología de análisis y una serie de indicadores que nos permitan conocer el avance y el impacto real que la utilización de la e-aula ha supuesto en la mejora de la calidad de la docencia, o al menos como ha sido percibida por parte del alumnado. En el curso académico 2005/06 se elaboró un cuestionario enfocado a conocer el impacto que había tenido en la docencia la aplicación del apoyo virtual. Se llevaron a cabo las siguientes preguntas, pidiendo en todas ellas una valoración cuantitativa en una escala de 1 a 10: 1) Transparencias de clase, 2) Información relacional, 3) Transmisión de la información, 4) Presentación de las prácticas, 5) Comunicación con los profesores, y 6) Valoración global. La encuesta se realizó por separado a los alumnos de agrónomos y montes para facilitar el posterior análisis de los resultados.

El análisis de las encuestas se ha llevado a cabo en una doble dirección: 1) conocer la valoración global y pormenorizada de los principales apartados de la aplicación y contrastar los resultados obtenidos con las consideraciones que sobre los aspectos específicos tienen los profesores como elaboradores de la aplicación. 2) Clasificar la respuesta del alumnado en grupos y contrastar las respuestas con las calificaciones obtenidas, relacionadas con el esfuerzo y por lo tanto con la 
utilización de la aplicación. A partir de contrastar resultados de la encuesta y resultados académicos, a través de un modelo podremos conocer el valor estimado de calificaciones en futuros cursos a partir de los valores de la encuesta (Sierra, 1994).

Para alcanzar el primero de los objetivos se ha llevado a cabo un análisis descriptivo de los mismos. Para el segundo caso se ha utilizado una técnica estadística dentro de las del análisis multivariante como es el Análisis Factorial Discriminante (FDA) (Aciego et al., 2005; Del Barrio et al., 2006) para llevarlo a cabo se ha utilizado el programa estadístico SPSS de acuerdo a Pérez (2001) se trata de un programa de fácil manejo para este tipo de análisis multivariante por su amigable entorno.

\section{RESULTADOS Y DISCUSIÓN}

\section{Resultados descriptivos}

El análisis descriptivo de los resultados de la encuesta tanto por separado para las dos titulaciones como conjunto señalan un más que aceptable aprobación de la misma. En la figura 2, se muestran los resultados obtenidos. Destacar la homogeneidad de los mismos, tanto entre titulaciones como para cada uno de los ítems analizados. Destaca asimismo que el valor mínimo en todos los casos se sitúa en 4 y las desviaciones típicas para cada ítem se sitúan en valores entre 1,42 y 1,91. El valor del coeficiente de curtosis se sitúa en valores próximos a -1. Como se observa, son las transparencias, esto es la documentación utilizada por el profesorado para las clases de teoría y las explicaciones de las prácticas, el aspecto más valorado, superando de media el valor de los 8 puntos. Esta circunstancia se fundamenta en que esa documentación va a ser utilizada directamente para la realización de las pruebas teóricas de la asignatura y los alumnos valoran muy positivamente su disponibilidad en formato digital para la consulta y el estudio.

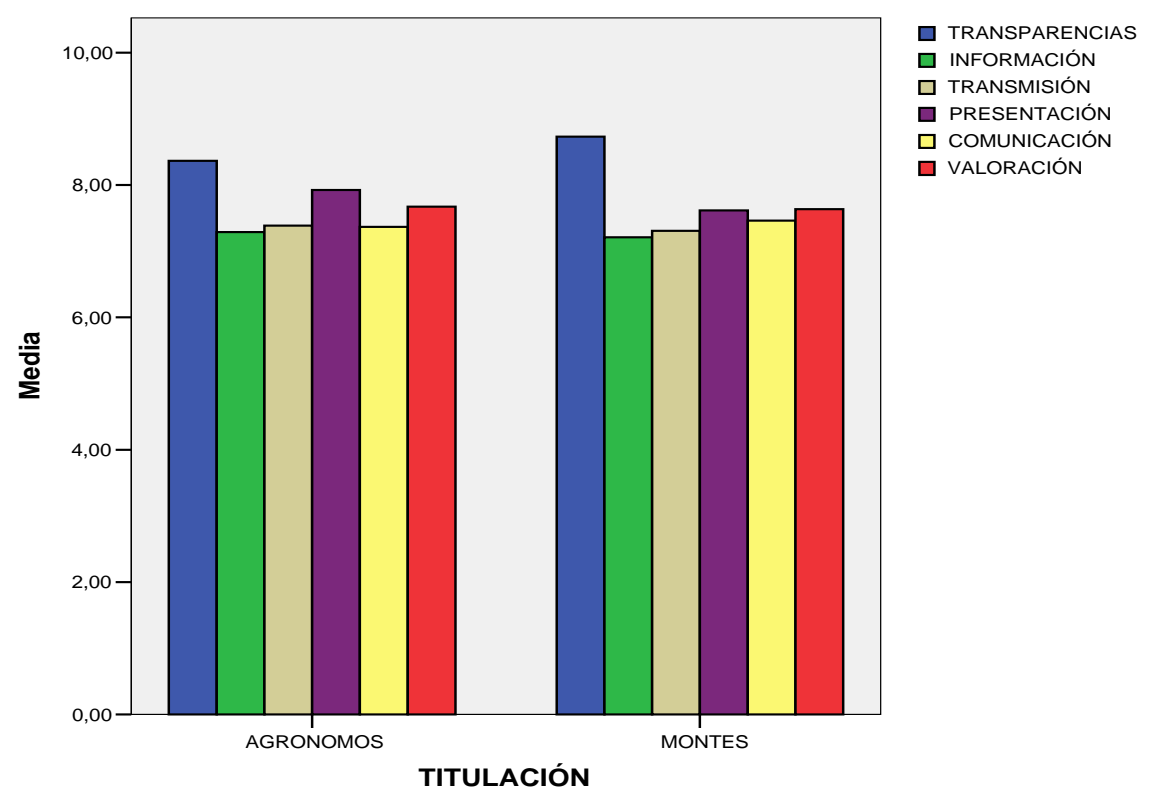

Fig. 2: Resultados para las diferentes preguntas y titulaciones

\section{Resultados del análisis multivariante (FDA)}

Dando respuesta al segundo de los objetivos se lleva a cabo un análisis factorial discriminante (FDA), que nos permita clasificar los alumnos en función de las calificaciones aportadas a la eaula de proyectos, en relación a superar o no la asignatura y posteriormente a la calificación obtenida en la asignatura. Con relación a superar la asignatura, se observa en la tabla 1, que existen diferencias en lo que se refiere a los valores medios de las calificaciones para las diferentes preguntas. Como se observa se producen diferencias en lo referentes a los valores de centralización y dispersión medios para cada una de las preguntas analizadas. En ambos casos, son la información de consulta para el estudio, las transparencias, la más valorada. Se produce la 
circunstancia de ser más valorada por los alumnos que no han superado el examen que por aquellos que lo han superado. A su vez la valoración global es ligeramente superior en el caso de los alumnos que han superado la asignatura.

Como se observa en la tabla 2, en que se hace mención a las pruebas de igualdad de medias, la Lambda de Wilks pone de manifiesto que no existen diferencias entre grupos, es decir, nos encontramos con valores muy próximos a la unidad y el nivel de significación supera, en todos los casos por mucho el valor de referencia de 0,05. Se contrasta la homogeneidad de respuesta. A través del test de la M`cox se puede conocer la igualdad de la covarianzas entre las matrices de los grupos analizados. Si el nivel de significación se sitúa por debajo del valor de 0,10 no se puede rechazar la igualdad, en nuestro caso se sitúa en un valor de 0,215. A partir de este momento, se puede construir las funciones canónicas discriminantes que nos permitan construir nuestro modelo de clasificación. Se ha obtenido una sola función canónica, que explica el 100\% de la varianza y que presenta un valor de correlación canónica relativamente bajo, situándose en el 0,207. El test para la Landa de Wilks para las funciones canónicas es un valor de 0,632, muy por encima del valor de referencia de 0,10 , mostrando una vez más que no existen iferencias entre los grupos.

Tabla 1: Valores de centralización y dispersión para los aprobados y reprobados

\begin{tabular}{llll} 
& & Media & Desv.típ. \\
\hline \multirow{4}{*}{ Reprobado } & TRANSPARENCIAS & 8,7222 & 1,36510 \\
& INFORMACIÓN & 7,2222 & 1,56955 \\
& TRANSMISIÓN & 7,3333 & 1,86701 \\
& PRESENTACIÓN & 7,7500 & 1,71339, \\
& COMUNICACIÓN & 7,2500 & 1,57435 \\
& VALORACIÓN & 7,5000 & 1,27615 \\
\hline \multirow{4}{*}{ Aprobado } & TRANSPARENCIAS & 8,4559 & 1,46026 \\
& INFORMACIÓN & 7,2647 & 1,72443 \\
& TRANSMISIÓN & 7,3526 & 1,96054 \\
& PRESENTACIÓN & 7,7794 & 1,67373 \\
& COMUNICACIÓN & 7,500 & 1,83254, \\
& VALORACIÓN & 7,7353 & 1,38884, \\
\hline \multirow{5}{*}{ Total } & TRANSPARENCIAS & 8,5481 & 1,42706 \\
& INFORMACIÓN & 7,2500 & 1,66489 \\
& TRANSMISIÓN & 7,3462 & 1,92959 \\
& PRESENTACIÓN & 7,7692 & 1,67928 \\
& COMUNICACIÓN & 7,4135 & 1,74384 \\
\hline \hline & VALORACIÓN & 7,6538 & 1,34936 \\
\hline
\end{tabular}

Tabla 2: Pruebas de la igualdad de las medias de los grupos

\begin{tabular}{|llllllll|}
\hline & $\begin{array}{l}\text { Lambda } \\
\text { de Wilks }\end{array}$ & $\mathbf{F}$ & $\mathbf{g} / \mathbf{1}$ & $\mathbf{g} / \mathbf{2}$ & Sig. \\
\hline TRANSPARENCIAS & 0,992 & 0,818 & 1 & 102 & 0,368 \\
INFORMACIÓN & 1,000 & 0,015 & 1 & 102 & 0,902 \\
TRANSMISIÓN & 1,000 & 0,002 & 1 & 102 & 0,961 \\
PRESENTACIÓN & 1,000 & 0,007 & 1 & 102 & 0,993 \\
COMUNICACIÓN & 0,995 & 0,481 & 1 & 102 & 0,489 \\
VALORACIÓN & 0,993 & 0,714 & 1 & 102 & 0,400 \\
\hline \hline
\end{tabular}

A partir de los coeficientes estandarizados de las funciones discriminantes canónicas obtenemos las funciones de los centroides de los grupos. A partir de este momento se repite la prueba de Box, pero en este caso para las funciones canónicas y calculamos las probabilidades previas para los grupos. Se establecen los coeficientes de las funciones de clasificación de acuerdo a las funciones discriminantes lineales de Fisher, que se muestran en la Tabla 3. 
Tabla 3: Coeficientes de la función de clasificación

\begin{tabular}{lll}
\hline & reprobado & aprobado \\
\hline TRANSPARENCIAS & 3,134 & 2,817 \\
INFORMACIÓN & 0,421 & 0,343 \\
TRANSMISIÓN & $-0,507$ & $-0,549$ \\
PRESENTACIÓN & 1,267 & 1,196 \\
COMUNICACIÓN & 1,159 & 1,114 \\
VALORACIÓN & 0,119 & 0,658 \\
(Constante) & $-23,946$ & $-22,935$ \\
\hline \hline
\end{tabular}

En este caso para los alumnos reprobados la estimación de la clasificación se haría a partir de la siguiente ecuación:

3,134 Transparencias + 0,421 Información - 0,597 Transmisión + 1,267 Presentación + 1,159 Comunicación $+0,119$ Valoración global $-23,946$. Análogamente se haría para cada uno de los alumnos aprobados por sus respectivos coeficientes. En la tabla 4 se muestran los resultados de la clasificación FDA. Como se observa se ha clasificado correctamente un $67,3 \%$ de los casos. Globalmente se puede considerar un buen resultado, pero conviene señalar que los problemas de errores en la clasificación se centran en los reprobados, que es en los que el porcentaje de errores se dispara, mientras que en los aprobados este se sitúa en casi un $95 \%$ de clasificados correctamente por el modelo.

Tabla 4: Resultados de la clasificación

\begin{tabular}{llllll} 
& & & \multicolumn{3}{c}{$\begin{array}{c}\text { Grupo de pertenencia } \\
\text { pronosticado }\end{array}$} \\
\hline & $\begin{array}{l}\text { Aprobados } \\
\text { reprobados }\end{array}$ & 0 & reprobado & aprobado & Total \\
\hline \multirow{3}{*}{ Original } & \multirow{2}{*}{ Recuento } & reprobado & 6 & 30 & 36 \\
& & aprobado & 4 & 64 & 68 \\
\cline { 3 - 6 } & reprobado & 16,7 & 83,3 & 100,0 \\
& aprobado & 5,9 & 94,1 & 100,0 \\
\hline \hline
\end{tabular}

Tabla 5: Resultados de la matriz de las funciones discriminante y las variables

\begin{tabular}{llll} 
& \multicolumn{3}{c}{ Función } \\
\cline { 2 - 4 } & $\mathbf{1}$ & $\mathbf{2}$ & $\mathbf{3}$ \\
\hline TRANSPARENCIAS & $0,523^{*}$ & $-0,046$ & 0,047 \\
PRESENTACIÓN & 0,511 & $0,628^{*}$ & 0,402 \\
INFORMACIÓN & 0,145 & $0,512^{*}$ & $-0,199$ \\
VALORACIÓN & $-0,016$ & $0,485^{\star}$ & 0,403 \\
COMUNICACIÓN & $-0,196$ & $-0,135$ & $0,634^{\star}$ \\
TRANSMISIÓN & 0,376 & 0,351 & $0,426^{\star}$ \\
\hline \hline
\end{tabular}

Tabla 6: Coeficientes de la función de clasificación para diferentes calificaciones

\begin{tabular}{lllll}
\cline { 2 - 5 } & \multicolumn{2}{l}{ Calificación } & & \\
\cline { 2 - 5 } & reprobado & aprobado & notable & sobresaliente \\
\hline TRANSPARENCIAS & 3,157 & 2,930 & 2,856 & 2,503 \\
INFORMACIÓN & 0,380 & 0,301 & 0,249 & 0,519 \\
TRANSMISIÓN & $-0,444$ & $-0,421$ & $-0,499$ & $-0,670$ \\
PRESENTACIÓN & 1,360 & 1,466 & 1,208 & 0,967 \\
COMUNICACIÓN & 1,110 & 0,955 & 1,200 & 0,947 \\
VALORACIÓN & 0,001 & 0,438 & 0,478 & 1,062 \\
(Constante) & $-23,868$ & $-24,812$ & $-23,245$ & $-23,131$ \\
\hline \hline
\end{tabular}


El mismo tipo de análisis se lleva a cabo, pero diferenciando entre los aprobados de acuerdo a la clasificación obtenida, (aprobado, notable y sobresaliente). De esta forma nos encontramos con 4 grupos en lugar de los dos que teníamos antes. A mayor número de grupos implica mayor complejidad del análisis y consecuentemente mayor nivel de error previsible (Rencher, 1998; Rosner, 2000). Se repiten los pasos del análisis. Los resultados siguen presentando una gran homogeneidad entre los grupos. Los valores identificados en la tabla 5 con * indican mayor correlación absoluta entre la variable y la función discriminante. Se obtienen los coeficientes de la función de distribución que se muestran en la tabla 6.

A partir de los datos de la tabla 5 y 6 se obtienen los resultados para los diferentes alumnos para las funciones discriminantes canónicas y los centros para cada uno de los grupos. Estos resultados se muestran en la figura 3. Se observa que hay diferencias entre los centroides de los grupos, muestra de la homogeneidad de respuesta comentada a lo largo de todo el trabajo. Por último se lleva a cabo la clasificación, de forma que se observen resultados desfavorables que para el caso de los dos grupos. El modelo sólo logra clasificar bien el $45,2 \%$ de los alumnos.

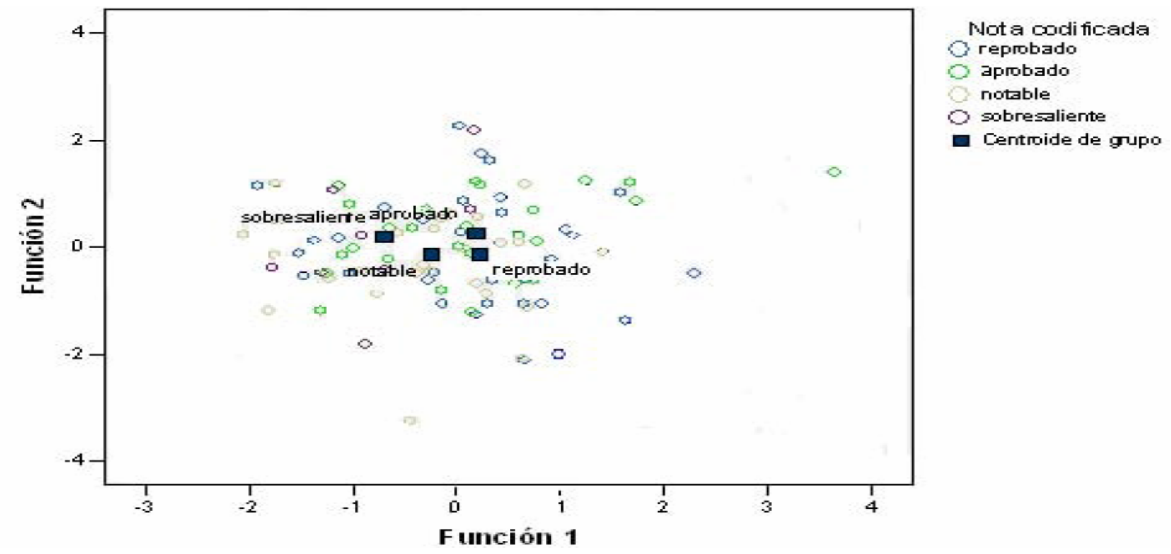

Fig. 3: Ubicación de los alumnos y los centroides de grupo

\section{CONCLUSIONES}

El desarrollo de aplicaciones virtuales de apoyo a la docencia universitaria supone un instrumento de gran importancia en la mejora de la misma. Las evaluaciones de estas aplicaciones permiten conocer el nivel de acierto en su desarrollo. En este trabajo se ha puesto de manifiesto que los alumnos consideran positivamente en general este tipo de herramientas, como ha demostrado el análisis descriptivo de las preguntas con el que conocemos la respuesta global del alumnado. La utilización de técnicas de clasificación multivariante constituye una herramienta muy interesante para enriquecer el análisis y poder conocer comportamientos futuros. En el caso analizado los resultados de la encuesta presentan una gran homogeneidad que limita las potencialidades del FDA, no pudiendo llevar un a cabo un análisis por grupos separados que permitiese conocer mejor como es el nivel de aceptación y de respuesta en las calificaciones obtenidas por los alumnos. Este hecho permitiría al profesorado de la asignatura de proyectos adelantarse en futuros cursos a las calificaciones de los alumnos una vez que fueran realizadas las encuestas de valoración del curso. Será necesario que, en futuros cursos, el profesorado desarrolle diferentes indicadores o mejoras en los cuestionarios de modo que sea posible discriminar para dar respuesta al objetivo de disponer de indicadores comunes que permita evaluar en todo momento la calidad de docencia que se imparte y se recibe.

\section{REFERENCIAS}

Aciego, R.; F. Domínguez y P. Hernández; Consistencia interna y estructura factorial de un cuestionario sobre autorrealización y crecimiento personal. Psicothema: (17), 134-142 (2005)

Álvarez, C.J., M.F. Marey y R. Ramos; Diagnóstico y perspectivas de los ingenieros agrónomos: el caso de la Comunidad de Galicia. Col. Ing. Agrónomos de Galicia, La Coruña, España (2003)

Arancibia, D.; De la educación a distancia a las comunidades de aprendizaje. Boletín de educación: (37), 9 -31 (2006) 
Bautista, G., F. Borges y A. Forés; Didáctica universitaria en entornos virtuales de enseñanzaaprendizaje. Editorial Narcea, Madrid, España (2006)

Bosco, A.; Las TIC en los procesos de convergencia europea y la innovación en la universidad: oportunidades y limitaciones. Aula abierta: (86), 3-22 (2005)

Cebrián, M.; Impacto de las tecnologías de la información y comunicación en la Universidad. Bordón. Revista de pedagogía: (56), 587-601 (2004)

Cervantes, L. y, M.D. Barrios; Nuevas tecnologías en educación. Ethos educativo: (33-34), 107131 (2005)

Del Barrio, M.V.; M.A. Carrasco y F.P.Holgado; Análisis transversal de los cinco factores de personalidad por sexo y edad de los niños españoles. Revista Latinoamericana de Psicología: 38(3), 567-577 (2006)

ERT; Education for Europeans. Towards the learning society. European Round Table for Industiralist. Bruselas, Bélgica (1995)

García, B.C. y T. Pinto; Las nuevas tecnologías de la información y comunicación y las funciones del profesor universitario. Encuentro Educacional: 12(3), 415-442 (2005)

González, J. y R. Wagenaar; Tuning Educational Structures in Europe. Informe Final. Fase Uno. Universidad de Deusto y Universidad de Groningen. Bilbao. España (2003)

Henao, O.; Evidencias de la investigación sobre el impacto de las tecnologías de información y comunicación en la enseñanza de la lecto-escritura. Rev. educación y pedagogía: (44), 71-83 (2006)

Hernández, F.; Los docentes y las TIC: cuatro tendencias o más. Cuadernos de pedagogía: (363), 66-70 (2006)

Lavilla, M.M., J. Leneveu y A. Salinas; La adaptación cognitivo-lingüística del alumno y del profesorado con un soporte pedagógico versus informático. Campo abierto: (25), 2532 (2006)

Maltempi, M.V. y M. Rosa; A avaliação vista sob o aspecto da educação a distancia. A valiação e políticas públicas em educação: (14), 50-72 (2006)

Manassero, M.A., A. Vázquez y J.A. Acevedo; Evaluación de las actitudes del profesorado respecto a los temas CTS: nuevos avances metodológicos. Enseñanza de las ciencias: 22(2), 299-307 (2004)

Marín, V.; Las nuevas tecnologías de la información y la comunicación y formación del profesorado universitario. Propuesta de un trabajo conjunto. Innov. educativa: (17), 149-168 (2007)

Nieto, S. y M.J. Rodriguez; Results convergence in two designs research-innovation in university teaching through the TIC. Revista Española de Pedagogía: 65(236), 27-48 (2007)

Pérez, C.; Técnicas Estadísticas con SPSS. Prentice Hall, Madrid, España (2001)

Rencher, A.C.; Methods of Multivariate Analysis, Volume 2, Multivariate Statistical Inference and Applications. John Wiley \& Sons. New York, USA. (1998)

Rosner, B.; Fundamentals of Biostatistics: study guide. Brooks/Cole, Pacific Grove. California, USA. (2000)

Salas, F.E.; Hallazgos de la investigación sobre la inserción de las tecnologías de la información y la comunicación (TIC) en la enseñanza: la experiencia de los últimos 10 años en los Estados Unidos. Educación: 29(2), 53-74 (2005)

Salcedo P., M.A. Pinninghoff J. y R. Contreras; Los estilos de aprendizaje en las plataformas de educación a distancia. Paideia: (40), 129-142 (2006)

Sierra, R.; Análisis Estadístico Multivariable. Teoría y Ejercicios. Paraninfo, Madrid, España (1994)

Tejedor, F.J. y A. García-Valcárcel; Competencias de los profesores para el uso de las TIC en la enseñanza. Análisis de conocimientos y actitudes. Rev. española de pedagogía: (233), 21-40 (2006)

Valverde, G.M. y M.H. Rivas; La educación y el nuevo escenario de las tecnologías de información y comunicaciones. Investigación educative: (15), 155-172 (2005) 